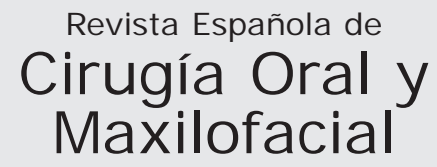

www.elsevier.es/recom

Caso clínico

\title{
Enfermedad por depósito de cristales de pirofosfato cálcico en la articulación temporomandibular con extensión intracraneal. A propósito de un caso
}

\author{
I. Zubillaga Rodríguez ${ }^{a, *}$, R. Gutiérrez Díaz ${ }^{a}$, G. Sánchez Aniceto ${ }^{b}$ y J.J. Montalvo Moreno ${ }^{c}$ \\ ${ }^{a}$ Médico Adjunto, Servicio de Cirugía Oral y Maxilofacial, Hospital Universitario 12 de Octubre, Madrid, España \\ befe de Sección, Servicio de Cirugía Oral y Maxilofacial, Hospital Universitario 12 de Octubre, Madrid, España \\ Jefe de Servicio, Servicio de Cirugía Oral y Maxilofacial, Hospital Universitario 12 de Octubre, Madrid, España
}

\section{INFORMACIÓN DEL ARTÍCULO}

Historia del artículo:

Recibido el 16 de junio de 2010

Aceptado el 21 de diciembre de 2010

Palabras clave:

Articulación temporomandibular;

Enfermedad por depósito de

cristales de pirofosfato cálcico;

Seudogota;

Cirugía de base de cráneo

\section{R E S U M E N}

Introducción: La articulación temporomandibular (ATM) humana es un conjunto de estructuras que permite la relación anatómica y funcional entre el hueso temporal y la mandíbula. Los síntomas y signos asociados a los procesos neoplásicos-seudotumorales de la ATM son similares a los asociados a los trastornos temporomandibulares.

Caso clínico: Presentamos un nuevo caso clínico de enfermedad por depósito de cristales de pirofosfato cálcico deshidratado (EDCPCD) con afectación de la ATM e invasión intracraneal. Se trata de una artropatía microcristalina que comparte características clínicas con la condromatosis sinovial. Se exponen los aspectos claves relativos al diagnóstico y el tratamiento de dicha patología.

Discusión: Los tumores que más frecuentemente afectan la ATM son las metástasis de tumores malignos. Entre las lesiones tumorales-seudotumorales que se originan directamente de la membrana sinovial se encuentran la condromatosis sinovial, la sinovitis villonodular, el sarcoma sinovial, la gota tofácea y la EDCPCD. Esta última es relativamente común en edades avanzadas, particularmente en la rodilla. El análisis de los cristales es esencial para establecer un diagnóstico de certeza. El tratamiento es eminentemente quirúrgico.

Conclusiones: La EDCPCD en la ATM es extremadamente rara. Las masas tumorales que surgen como tumores primarios en la ATM representan un problema diagnóstico desafiante. La variada presentación de estas lesiones a menudo enmascara la patología de base y conlleva su retraso en el diagnóstico.

( 2010 SECOM. Publicado por Elsevier España, S.L. Todos los derechos reservados.

\footnotetext{
*Autor para correspondencia.

Correo electrónico: izubillaga.hdoc@salud.madrid.org (I. Zubillaga Rodríguez).
} 
Keywords:

Temporomandibular joint; Calcium pyrophosphate deposition disease;

Pseudogout;

Skull base surgery
Calcium pyrophosphate deposition disease of the temporomandibular joint with intracranial extension. A case report

\begin{abstract}
A B S T R A C T
Introduction: The human temporomandibular joint (TMJ) is the structural complex that supports the functional and anatomic relations between the temporal bone and mandible. Symptoms and signs related to neoplastic or pseudotumoral disease of this joint are similar to the symptoms associated with temporomandibular joint dysfunction.

Case report: We report a new case of calcium pyrophosphate deposition disease (CPDD) of the TMJ with intracranial spread. CPDD is a microcrystaline arthropathy with some clinical characteristics of synovial chondromatosis. Key aspects of the diagnosis and treatment of CPDD are discussed.

Discussion: The most frequent tumors of the TMJ are metastatic. Among the tumoralpseudotumoral lesions that originate directly on the synovial membrane are synovial chondromatosis, villonodular synovitis, synovial sarcoma, tophaceous gout and CPDD. CPDD is relatively common in elderly patients, especially in the knee. Analysis of the crystals is essential for diagnosis. Surgical treatment is indicated.

Conclusions: CPDD is an extremely rare disease in the TMJ. TMJ tumor masses are a diagnostic challenge. The wide variety of presenting symptoms frequently obscures the underlying pathology and delays diagnosis.
\end{abstract}

(c) 2010 SECOM. Published by Elsevier España, S.L. All rights reserved.

\section{Introducción}

La articulación temporomandibular (ATM) humana, sinovial y bicondílea, es un conjunto de estructuras que permite la relación anatómica y funcional entre el hueso temporal y la mandíbula. Esta articulación permite realizar los movimientos mandibulares y las funciones del sistema estomatognático.

La patología de la ATM es similar a la de cualquier articulación del organismo, incluyendo anomalías congénitas y del desarrollo, traumatismos, artritis y neoplasias.

Los síntomas y signos asociados a los procesos neoplásicos-seudotumorales de la ATM son: aumento de tamaño preauricular, asimetría progresiva, maloclusión, dolor, clic o chasquido, restricción de movimientos mandibulares, limitación de la apertura oral, cambios auditivos y parestesias. Éstos son similares a los signos y síntomas asociados a los trastornos temporomandibulares, por lo que el diagnóstico de estas entidades es relativamente complejo.

La enfermedad por depósito de cristales de pirofosfato cálcico deshidratado (EDCPCD) es una artropatía microcristalina que comparte características clínicas con la condromatosis sinovial. La patogenia es desconocida; sin embargo, la sobreproducción de pirofosfato extracelular desempeña un papel fundamental. Desconocemos si el depósito de cristales por sí mismo altera y difunde a través del cartílago articular o si los traumatismos previos favorecen la erosión de los cristales a través de los límites de la articulación. La ATM es una localización rara para esta enfermedad, siendo más frecuentes la patología tumoral y metastásica. Clínicamente pueden simular inicialmente un síndrome de disfunción temporomandibular, y los hallazgos radiológicos varían dependiendo del grado de agresividad-osteólisis local. Es una rara artropatía metabólica erosiva caracterizada por depósito de cristales de calcio intra-periarticulares. Los síntomas más frecuentes cuando se encuentra afectada la ATM son dolor facial, otalgia, inflamación preauricular y trismo. Dependiendo de la naturaleza de los síntomas de presentación, el diagnóstico diferencial puede incluir neoplasias parotídeas, condromas, condrosarcomas, osteomas, colesteatomas y artropatías infecciosas.

El tratamiento es eminentemente quirúrgico. La exéresis completa de los depósitos de cristales es mandatoria, ya que la resección parcial puede potencialmente predisponer a la recurrencia de los síntomas.

\section{Caso clínico}

Paciente varón de 73 años que acude a consultas externas de cirugía oral y maxilofacial del Hospital Universitario 12 de Octubre por presentar tumefacción progresiva de crecimiento lento en la región preauricular derecha. El tiempo de evolución desde el inicio de la sintomatología es de 3 meses. Entre sus antecedentes personales destaca la presencia de hipertensión arterial controlada con tratamiento médico por vía oral e hipertrofia benigna de próstata con repercusión clínica pendiente de ser intervenida quirúrgicamente.

Refiere clínica insidiosa de dolor-disfunción en ATM derecha de aproximadamente 1 año de evolución, con limitación progresiva de la apertura oral. En el momento de su exploración clínica su máxima apertura oral era de $25 \mathrm{~mm}$. No se objetivan lesiones intraorales, signos neurológicos ni existe clínica en relación con alteración de pares craneales, incluido un VII par craneal clínicamente normal. La exploración cervical no presenta signos de interés.

Se decide solicitar una punción-aspiración con aguja fina (PAAF) de la región preauricular parotídea derecha, congruente con adipocitos maduros sobre tejido glandular salivar a este nivel. 


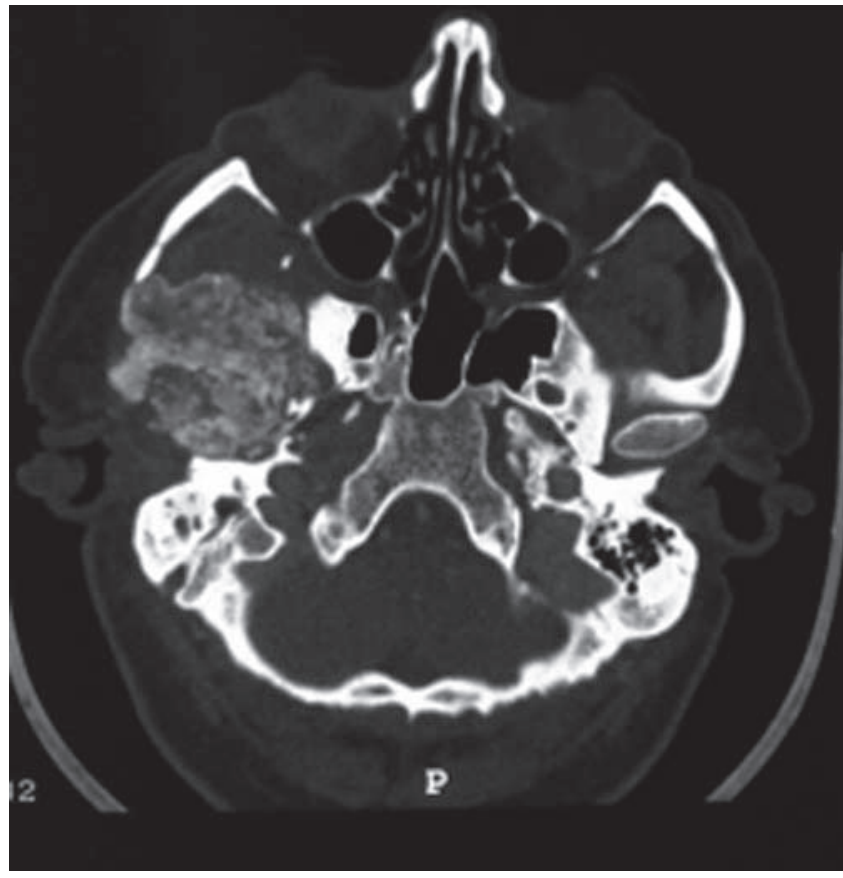

Figura 1 - Tomografía computarizada (TC) preoperatoria (corte axial).

Dentro de las pruebas de imagen preoperatorias se incluye una TC facial, en la que se objetiva una lesión polilobulada con desplazamiento condilar e invasión de la base del cráneo con áreas de anillos y semicírculos que sugiere el diagnóstico de condrosarcoma de ATM derecha (fig. 1).

En la RM de macizo facial se identifica una masa en torno a la ATM derecha sin destrucción del cóndilo mandibular, heterogénea, con osteólisis de la base del cráneo en la región del ala mayor del esfenoides y con un pequeño componente epidural. Existe una captación de contraste tras la administración del mismo, excepto en las zonas de calcificación. Dichas calcificaciones se localizan en el espacio masticatorio (fig. 2). Las características de la imagen sugieren alta agresividad, y hay que valorar la posibilidad de osteo-condrosarcoma.

Ante la afectación de la base craneal se decide solicitar una TC específica de dicha región anatómica. Existe una lesión que crece en la fosa temporal e infiltra el ala mayor del esfenoides derecho sin afectar la porción basal del peñasco. La lesión en el momento actual tiene un tamaño de $45 \times 22 \times$ $38 \mathrm{~mm}$. Crece fundamentalmente hacia la fosa temporal más que hacia el espacio intracraneal. El tumor puede iniciarse en la cavidad glenoidea del peñasco. Llama la atención en su crecimiento el hecho de que afecta y parece desplazar el cóndilo mandibular hacia caudal.

El tumor no parece afectar la yugular ni la carótida, y a nivel del peñasco estas estructuras se ven con normalidad. En conclusión, hay tumoración de la estructura condroide, sin poder descartar grado de malignidad o degeneración sarcomatosa de la misma.

Ante la discrepancia clínico-radiológica tras las pruebas complementarias solicitadas y dada la posibilidad de tratarse de una lesión con alto grado de malignidad tras los informes

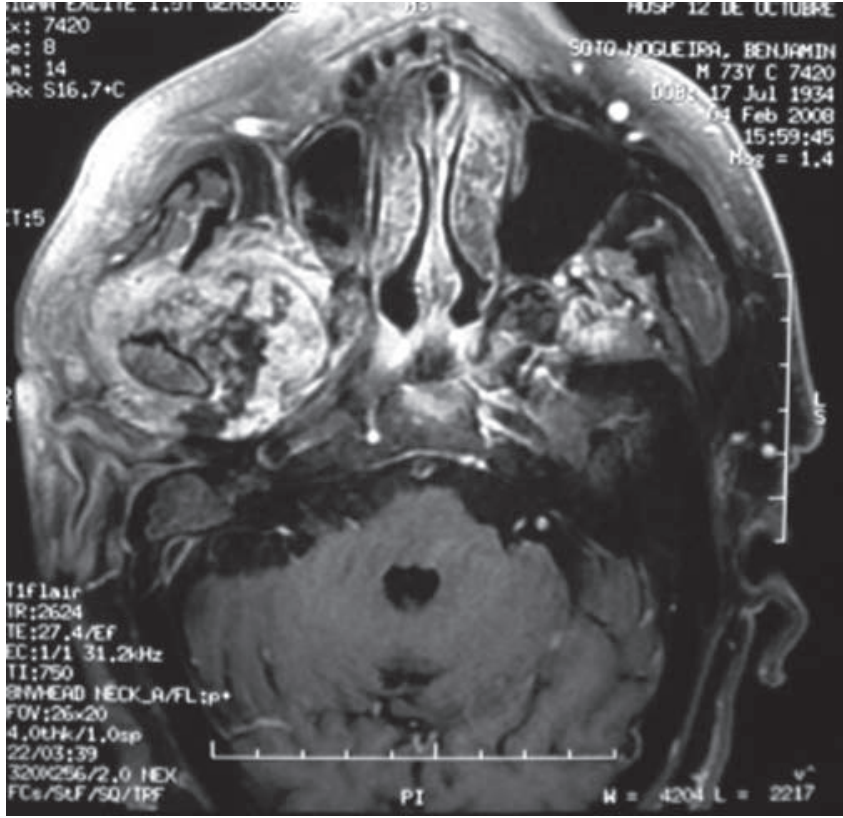

Figura 2 - Resonancia magnética (RM) preoperatoria (corte axial).

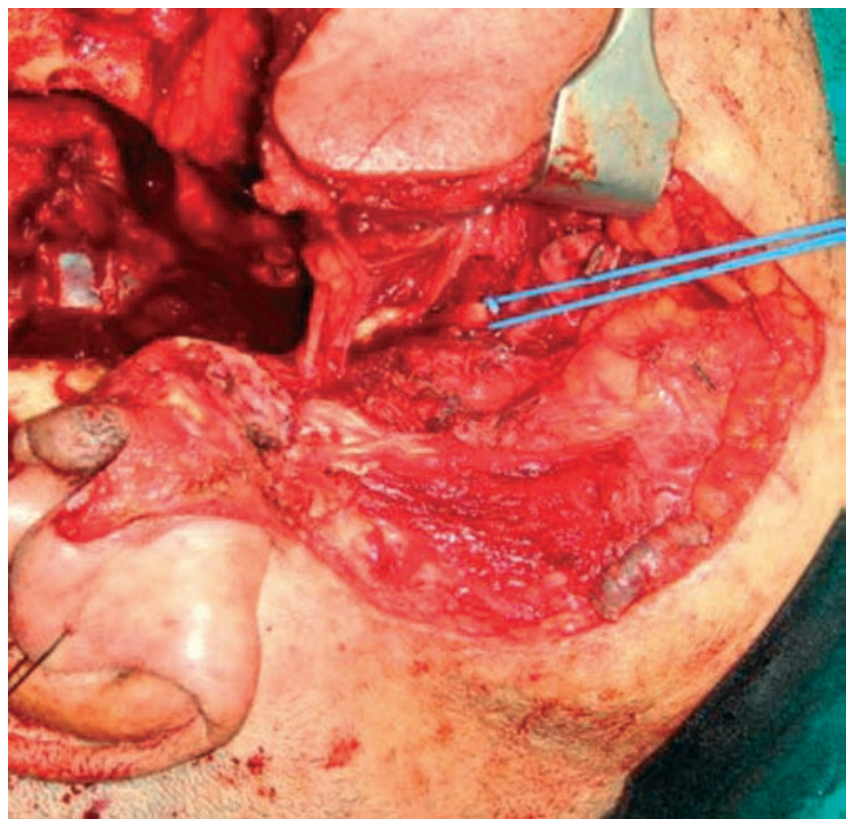

Figura 3 - Vessel-loop con control de la arteria carótida interna a nivel cervical.

radiológicos, se decide, en colaboración con el Servicio de Neurocirugía, la exéresis tumoral en bloque mediante abordaje anterolateral a la base craneal.

El procedimiento quirúrgico implica la exéresis tumoral mediante un abordaje subtemporal-infratemporal, incluyendo craneotomía temporal basal derecha con disección epidural de la fosa media (sin infiltración intradural), orbitotomía lateral, preservación del VII par y control de la arteria carótida interna (fig. 3) con ligadura de la arteria carótida 


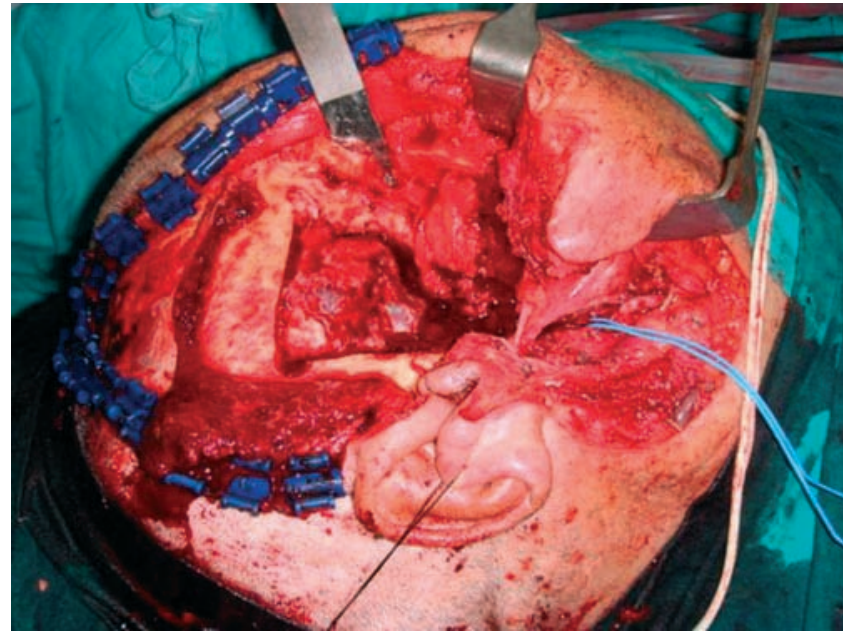

Figura 4 - Lecho quirúrgico post-resección.

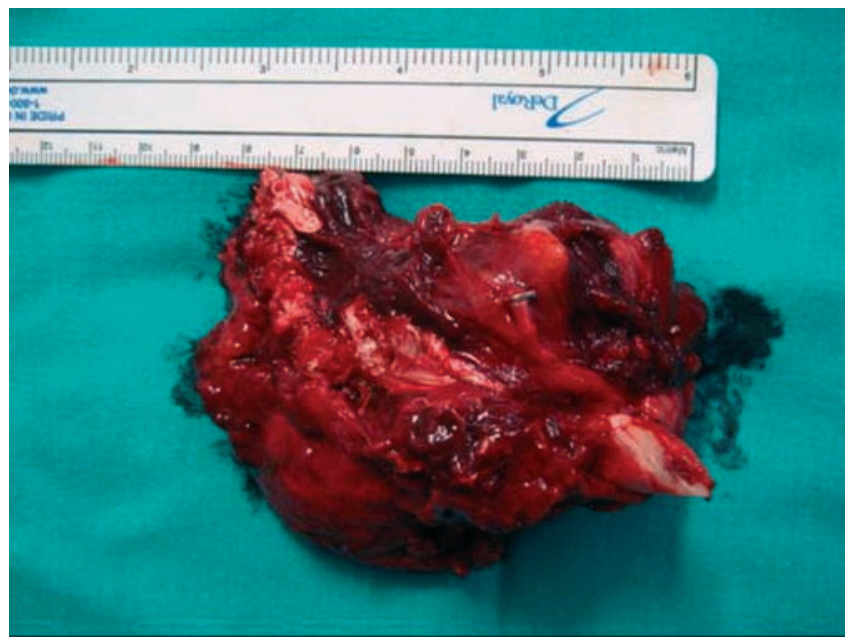

Figura 5 - Espécimen quirúrgico en bloque, incluyendo vaciamiento infratemporal y cóndilo mandibular.

externa (fig. 4). El espécimen quirúrgico incluye base temporal, arco cigomático, cóndilo mandibular y vaciamiento infratemporal (fig. 5). Se remiten intraoperatoriamente una adenopatía parotídea y otra cervical de nivel II informadas como sin evidencia de metástasis. La reconstrucción del defecto-espacio muerto de la base lateral fue realizada con un colgajo libre microquirúrgico anterolateral de muslo con dos perforantes (fig. 6). Se fija la craneotomía-orbitotomía con miniplacas de 1,3 mm (fig. 7). Se desepiteliza el colgajo manteniendo un tutor cutáneo que se sutura a nivel preauricular (fig. 8).

El examen anatomopatológico en su descripción macroscópica presenta una pieza de resección craneofacial lateral que mide 10,4 $\times 6,3 \times 5,4 \mathrm{~cm}$. En la pieza se identifica un tumor aparentemente bien delimitado, de contorno irregular, que mide $4 \times 5,3 \times 2,7 \mathrm{~cm}$. Al corte presenta una coloración heterogénea con mezcla de áreas blanquecinas y áreas parduzcas. Respecto a la descripción microscópica, revela la presencia de tejido fibroconectivo con una lesión con estroma fibromixoide, en la que hay abundantes islotes de material

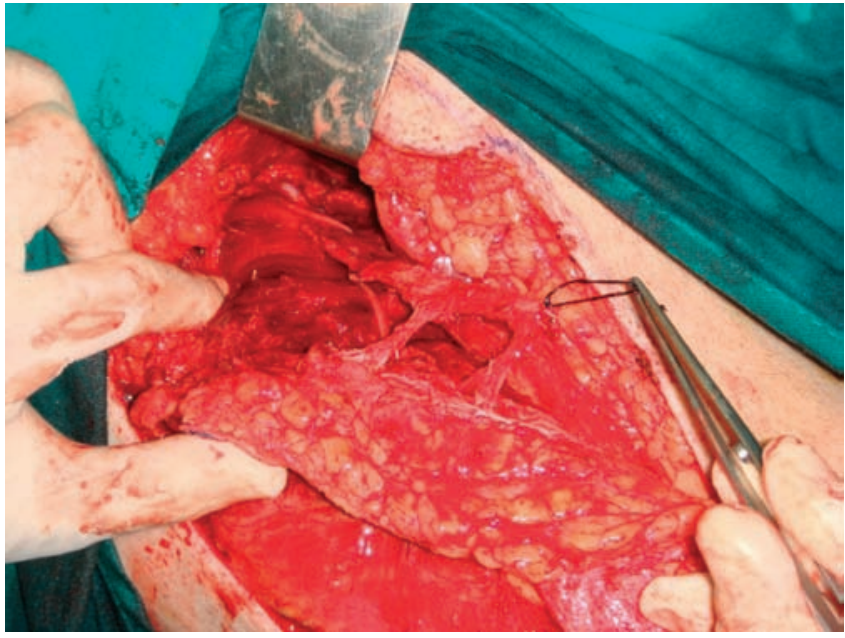

Figura 6-Tallado de colgajo anterolateral con dos perforantes.

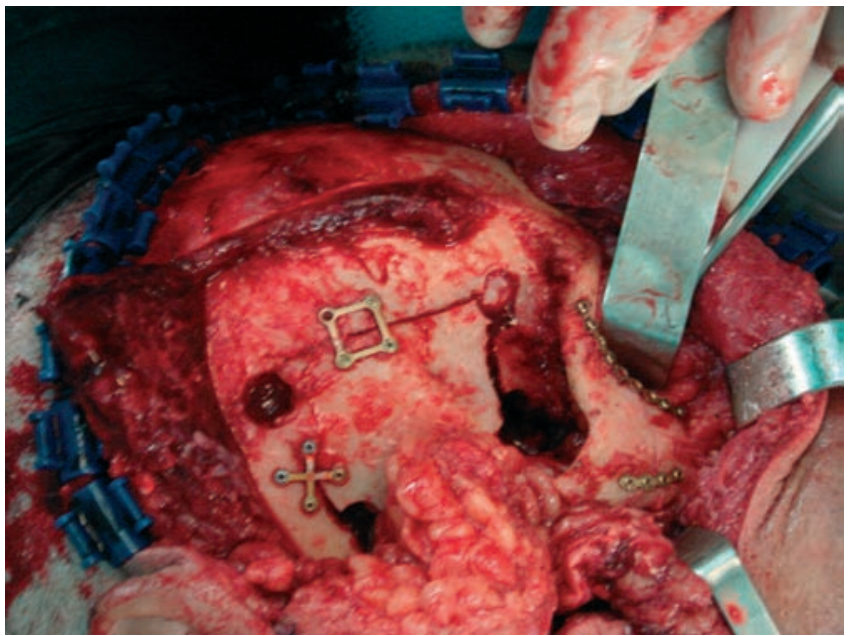

Figura 7-Osteosíntesis en craneotomía temporal-osteotomía orbitocigomática.

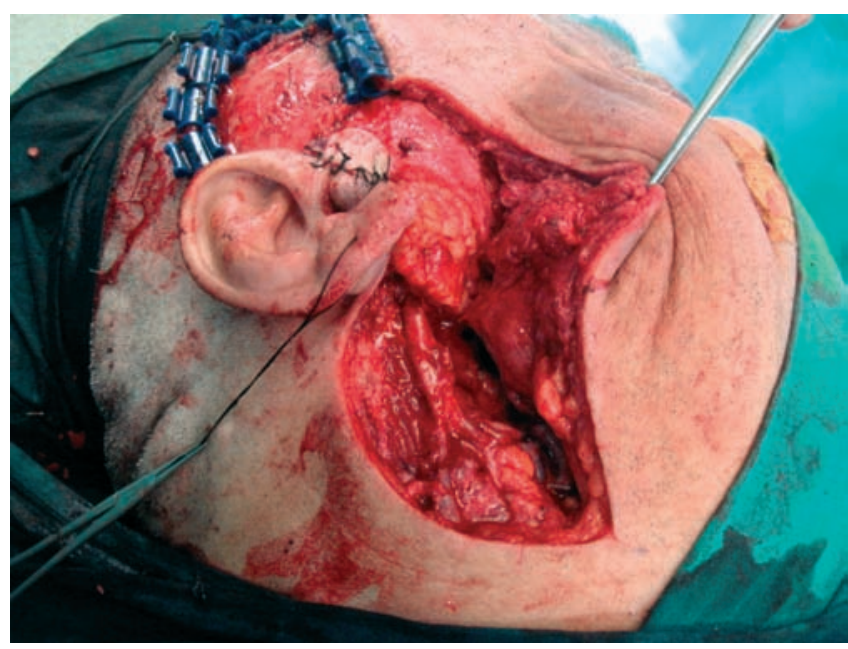

Figura 8 - Obliteración de la base craneal lateral con colgajo anterolateral. Se aprecia tutor cutáneo preauricular. 


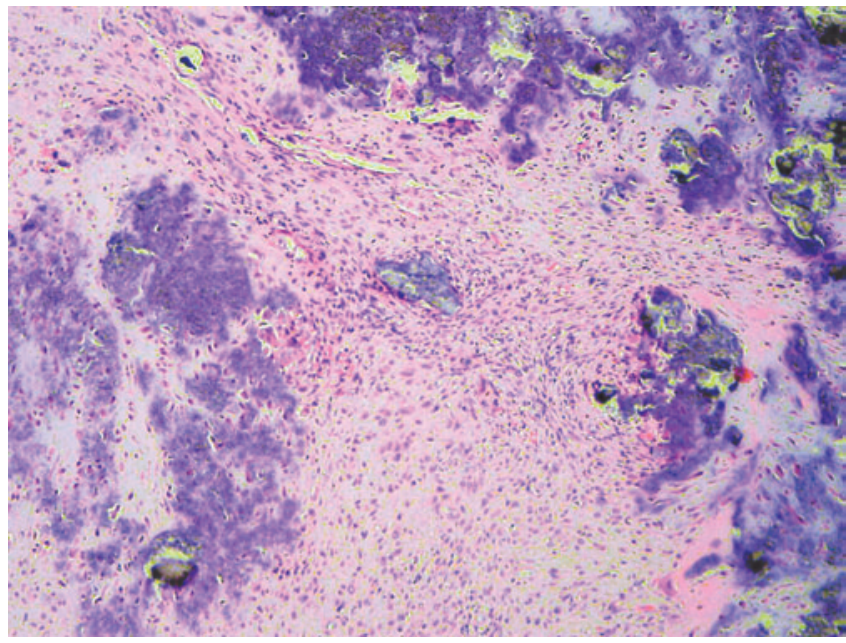

Figura 9 - Cristales aciculados con áreas calcificadas rodeados de denso estroma fibroso.

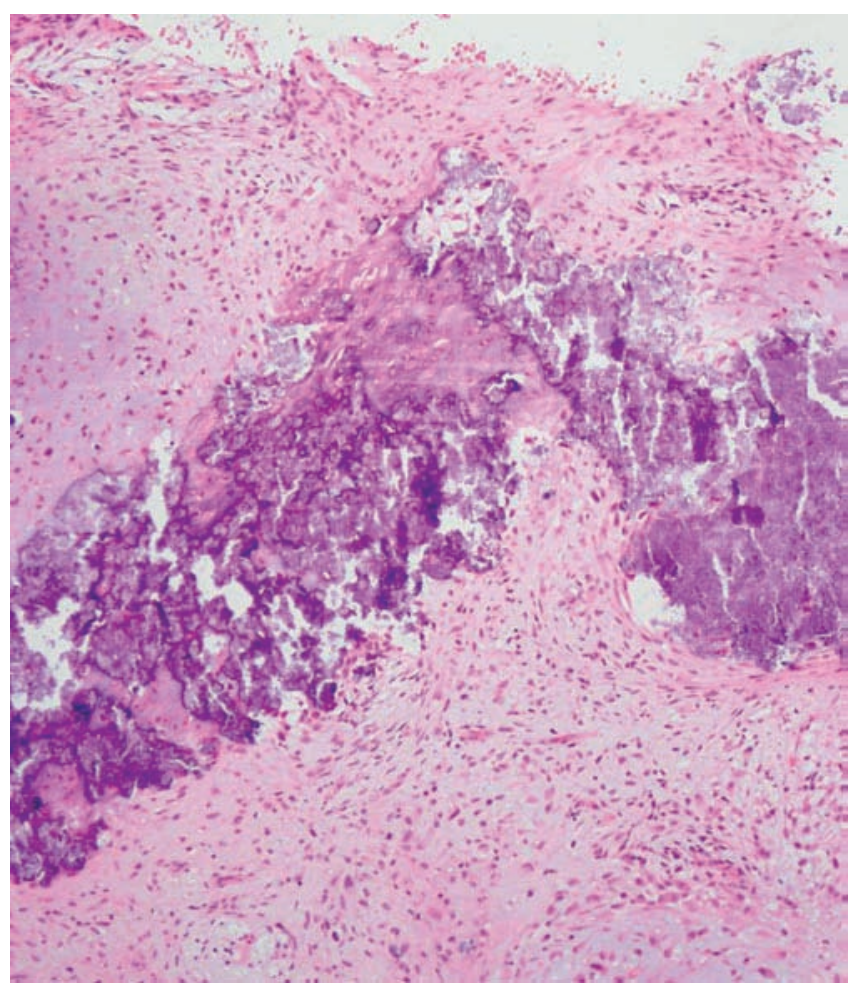

Figura 10 - Metaplasia condroide con atipia significativa asociada a cristales débilmente birrefringentes y células gigantes multinucleadas.

cristalino basófilo, con cristales aciculados, que birrefringen débilmente bajo luz polarizada (fig. 9). Alrededor de los cristales hay una gran cantidad de histiocitos y una intensa reacción gigantocelular a cuerpo extraño. También se observa metaplasia condroide con condrocitos con atipia significativa (fig. 10). La lesión no alcanza los extremos de resección, y el cóndilo mandibular está respetado. El estudio inmunohistoquímico presenta proliferación celular con fenotipo histioci-

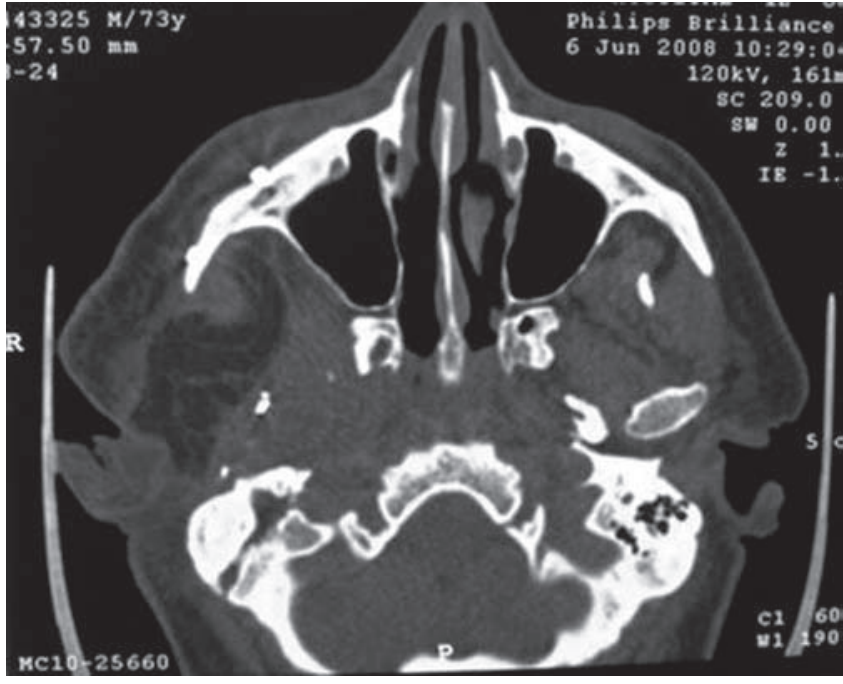

Figura 11 - Tomografía computarizada (TC) de control en la que se objetiva la obliteración de la fosa infratemporal con el colgajo anterolateral.

tario macrofágico y fibroblástico (CD 45-68+) con focos de metaplasia condral (S-100+).

El examen anatomopatológico muestra una pieza de resección craneofacial lateral con una lesión producida por depósito de pirofosfato cálcico deshidratado, con intensa reacción a cuerpo extraño, fibrosis y metaplasia condroide con atipia. La birrefringencia débil bajo luz polarizada diagnostica la EDCPCD.

El postoperatorio cursa sin complicaciones. Se realiza TC de control con adecuada posición del colgajo en la fosa infratemporal, sin evidencia de colecciones (fig. 11). En la RM de control se aprecia la exéresis completa y obliteración del espacio muerto en la base lateral (figs. 12-13).

El paciente evoluciona favorablemente, presentando una máxima apertura oral de $45 \mathrm{~mm} 3$ meses después de la cirugía (figs. 14-16). A los 6 meses el paciente desarrolla una lesión seudoartrósica en la articulación interfalángica en el dedo meñique de la mano derecha, y tras el estudio en el servicio de reumatología se objetiva relación con la EDCPCD (fig. 17). Dos años después de la cirugía el paciente se encuentra libre de enfermedad.

\section{Discusión}

Basándose en una revisión de la literatura, los tumores que más frecuentemente afectan la ATM son las metástasis de tumores malignos ${ }^{1}$. Si nos referimos a lesiones primarias, pueden potencialmente originarse del hueso, del cartílago o de la membrana sinovial. Los tumores óseos y cartilaginosos son más comunes que los sinoviales. Se han descrito osteocondromas, osteoblastomas, condroblastomas, condrosarcomas y neurofibromas. Entre las lesiones tumorales-seudotumorales que se originan directamente de la membrana sinovial se encuentran la condromatosis sinovial, la sinovitis villonodular, el sarcoma sinovial, la gota tofácea y la EDCPCD. 


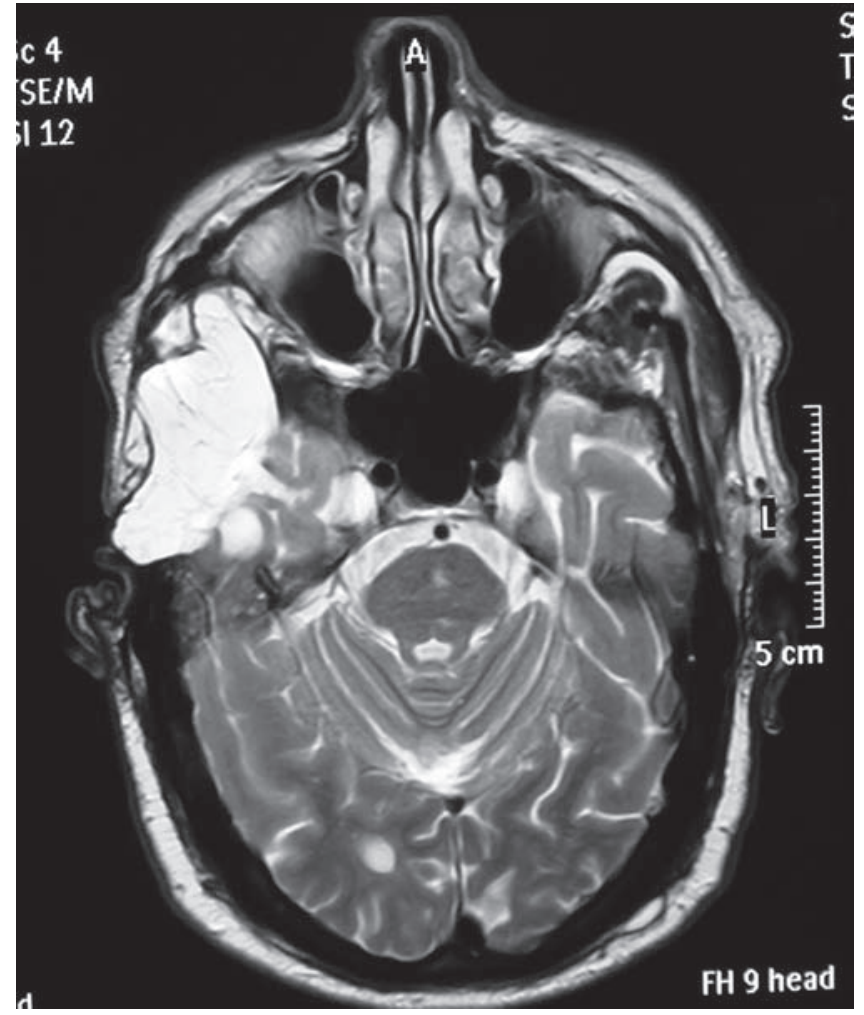

Figura 12 - Resonancia magnética (RM) de control (corte axial).

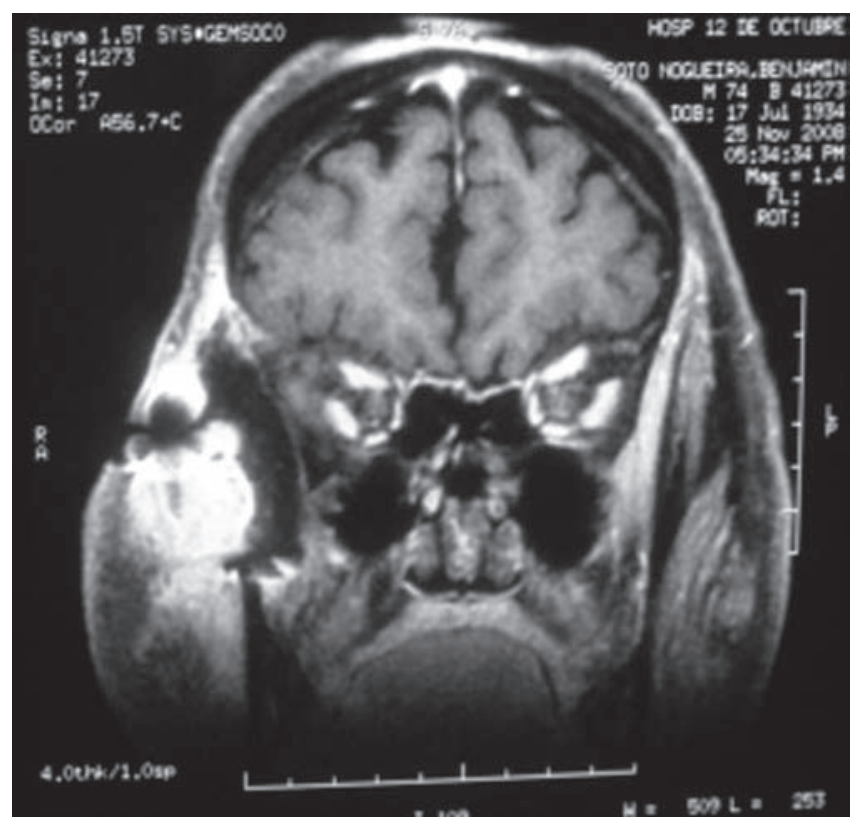

Figura 13 - Resonancia magnética (RM) de control (corte coronal).

La EDCPCD es relativamente común en edades avanzadas, particularmente en la rodilla. Los síntomas clínicos presentan un variado espectro, pero son similares a los que aparecen en las enfermedades reumatológicas. En 1962 McCarthy et al ${ }^{2}$

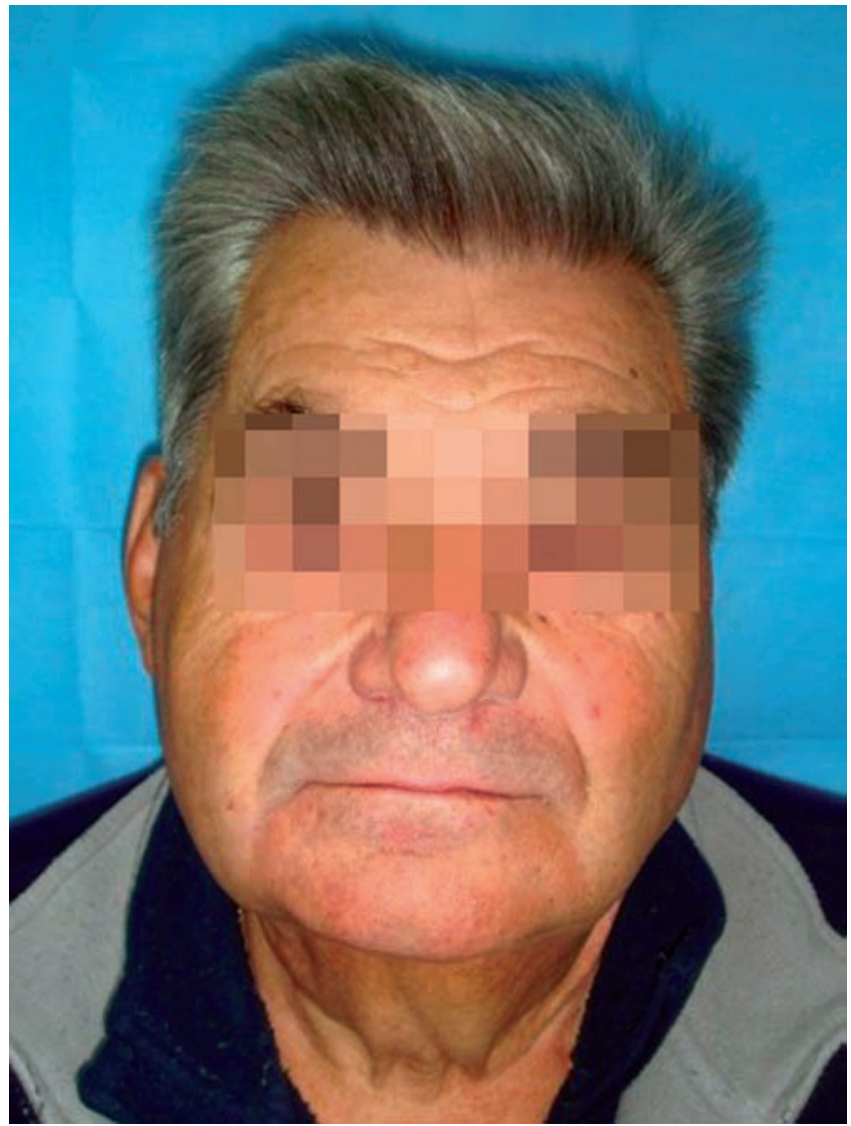

Figura 14-Control clínico a los 3 meses. Vista anteroposterior.

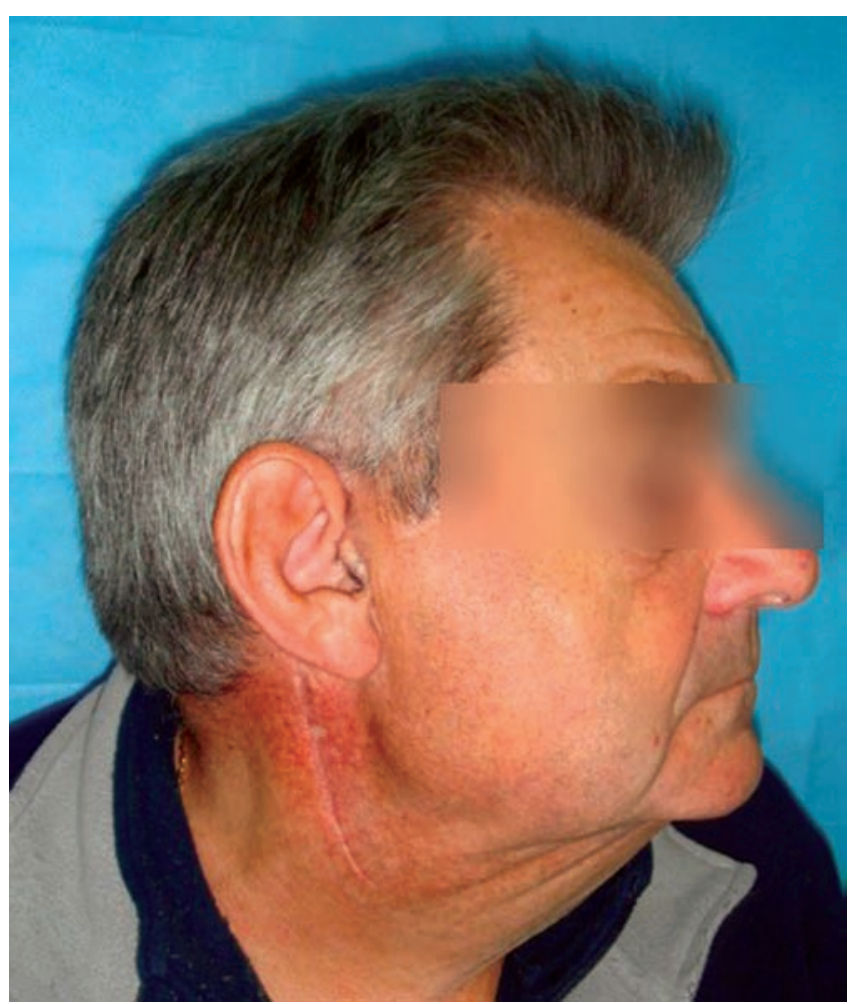

Figura 15 - Control clínico a los 3 meses. Vista lateral. 


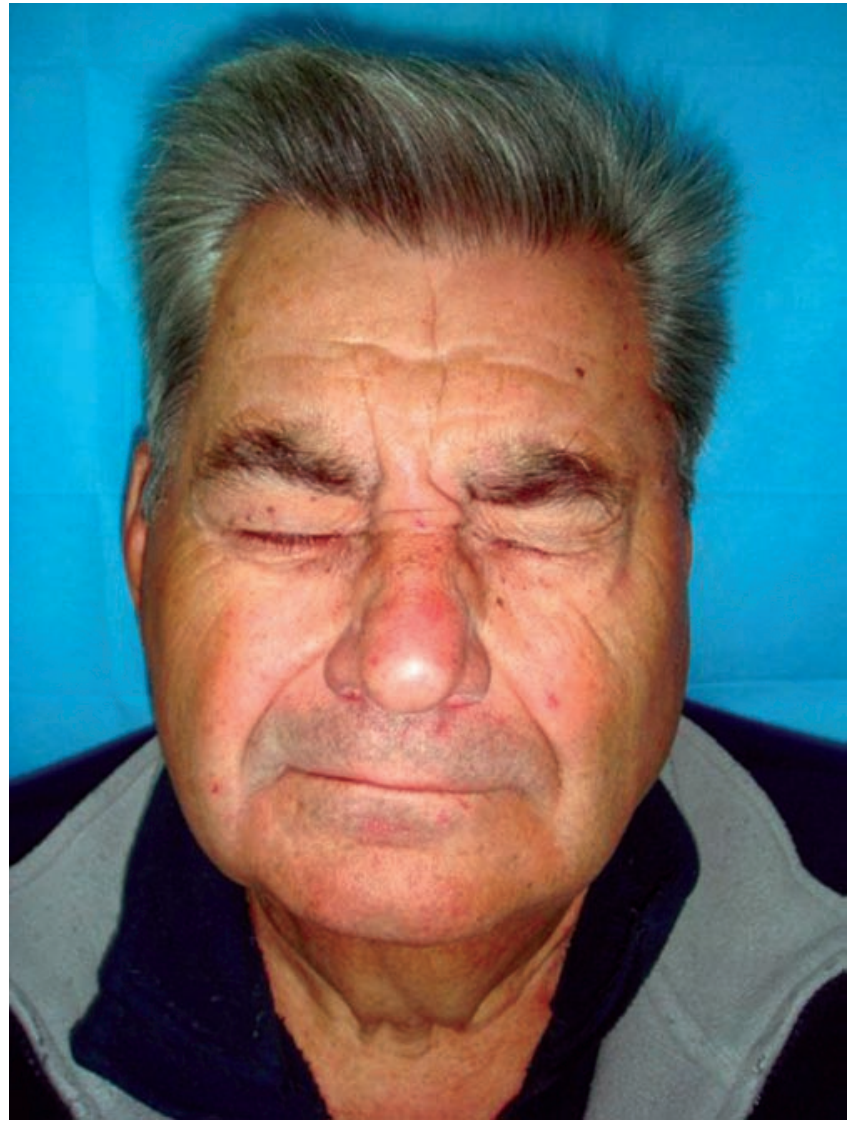

Figura 16 - Funcionalidad conservada del nervio facial.

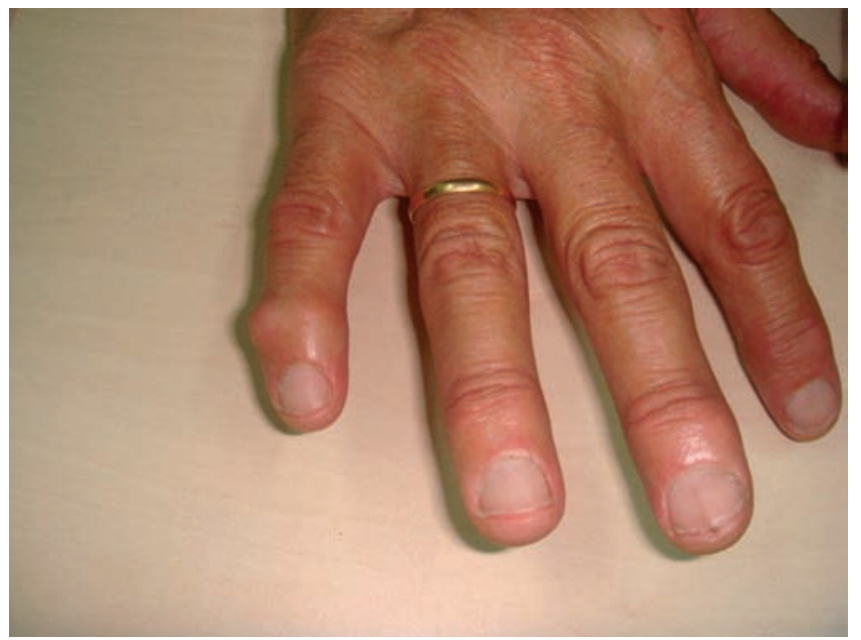

Figura 17 - Detalle de la afectación del dedo meñique de la mano derecha.

fueron los primeros en identificar cristales de pirofosfato cálcico deshidratado en el líquido sinovial de pacientes con síntomas similares a la gota tofácea (seudogota). Zitnan y Sitag ${ }^{3}$ aplicaron el término "condrocalcinosis" a la patología con características radiográficas específicas en las articulaciones durante ataques de artritis inflamatorias agudas. Como los cristales de pirofosfato cálcico deshidratado pueden presen- tar varias manifestaciones clínicas, Ryan et al propusieron el nombre de "enfermedad por depósito de cristales de pirofosfato cálcico deshidratado" como término general ${ }^{3}$.

Su aparición en la ATM es extremadamente rara. Además de la rodilla, otras articulaciones que pueden resultar afectas son las caderas, los hombros, los codos y los tobillos. Algunos estudios ${ }^{4}$ han mostrado un incremento en la prevalencia de esta enfermedad con la edad. Cuando la enfermedad se manifiesta en la ATM, el dolor y la disfunción articular pueden conducir a un diagnóstico más precoz que en otras articulaciones, aunque en la práctica clínica esta sintomatología puede enmascarar realmente la patología subyacente.

La EDCPCD ha sido clasificada etiológicamente en cuatro categorías: hereditaria, esporádica, asociada a otra enfermedad metabólica y asociada a traumatismos o cirugías previas. Los síntomas clínicos son variables y se asemejan a otras enfermedades articulares, como la gota ${ }^{5}$, la artritis reumatoide y la osteoartritis. Es realmente complicado diferenciar dichas entidades exclusivamente con los hallazgos clínicos. La gota es una enfermedad común, con hallazgos radiográficos clásicos. La alteración bioquímica que la produce es la hiperuricemia. El tofo gotoso representa la fase crónica de la enfermedad. La artritis gotosa es la forma más común de enfermedad articular inflamatoria en mayores de 40 años, y el diagnóstico se establece determinando niveles de urato sódico. Los hallazgos por técnicas de imagen pueden ser superponibles a los de la EDCPCD, y el diagnóstico definitivo requiere el examen del líquido sinovial que confirme la presencia de cristales de urato monosódico monohidratado (birrefringencia negativa bajo luz polarizada).

Entre las formas de presentación clínica de la EDCPCD se encuentran las formas destructivas, seudoartríticas, seudotumorales y seudogotosas. A esta última variante se la ha llamado seudogota tofácea, y hasta el año 2000 sólo se habían descrito 29 casos en todas las articulaciones ${ }^{6}$. Curiosamente, aunque la EDCPCD raramente afecta la ATM, es la articulación más frecuentemente afectada en su variante seudotumoral. Los síntomas más frecuentemente encontrados son la presencia de una masa indolora y edema preauricular.

Las masas tumorales que surgen como tumores primarios en la ATM son raras y representan un problema diagnóstico desafiante. La variada presentación de estas lesiones a menudo enmascara la patología de base y conlleva su retraso en el diagnóstico. A menudo se presentan como edema facial unilateral, obstrucciones del canal auditivo externo y disfunciones de la ATM. La persistencia de esta sintomatología nos obliga a recurrir a pruebas de imagen que puedan descartar patología neoplásica-seudotumoral subyacente. A pesar de esto, algunos pacientes no presentan un diagnóstico correcto hasta su examen histológico.

El desarrollo en las últimas décadas de técnicas de imagen más precisas ha contribuido al incremento en el diagnóstico de tumores asintomáticos de forma casual. En relación con dichas técnicas de imagen, la lesión puede manifestarse como un incremento del espacio articular, irregularidades de las superficies articulares, presencia de calcificaciones o esclerosis, e hiperostosis si existe afectación de la fosa glenoidea. La TC y la RM han demostrado ser los procedimientos diagnósticos más específicos. La TC puede determinar las 
áreas de calcificaciones y la erosión ósea causada por la lesión. La RM define mejor la anatomía del disco y de la cápsula articular, a la vez que visualiza los límites de la lesión detectando implicación de estructuras vecinas, especialmente si existe afectación hacia la base craneal ${ }^{7}$.

Aunque el diagnóstico de EDCPCD puede ser sugerido por los hallazgos radiológicos, el diagnóstico final se establece tras la evaluación histológica y la evidencia de birrefringencia débil de cristales romboidales bajo luz polarizada. Se detectan nódulos cartilaginosos con grados variables de cristalización. La sensibilidad-especificidad que alcanza la PAAF como prueba complementaria preoperatoria no es significativa.

El análisis de los cristales es esencial para establecer un diagnóstico de certeza. Aunque los cristales son débilmente birrefringentes bajo luz polarizada, otros cristales, como los de oxalato cálcico, de esteroides sintéticos y del ácido etilendiaminotetraacético (EDTA) también son birrefringentes. El diagnóstico diferencial debe basarse en el análisis cuantitativo de los cristales o en la observación de la estructura del cristal $^{8}$. Para detectar histológicamente los cristales, el espécimen debe ser examinado sin descalcificación, porque en secciones descalcificadas los cristales se pierden y los hallazgos atípicos de condrocitos metaplásicos pueden conducir a un error histológico en el diagnóstico, simulando condrosarcomas, sinovitis villonodular pigmentada o condromatosis sinovial.

El tratamiento de elección es quirúrgico. La afectación de una única articulación, el diagnóstico desconocido y la gravedad de los síntomas clínicos hacen que el tratamiento quirúrgico sea el apropiado ${ }^{9}$. Es necesario, además, para el correcto diagnóstico histológico de la patología.

La cirugía de la base del cráneo ha experimentado una revolución en las dos últimas décadas debido al desarrollo de nuevos abordajes a esta compleja área anatómica. En los equipos multidisciplinarios dedicados al tratamiento de la patología de la base craneal han cambiado los parámetros de que hasta ahora se consideraba como "no resecable", creando cada vez defectos mayores en la base craneal ${ }^{10}$. Sin embargo, dicha revolución no hubiese sido posible sin nuevas técnicas de reconstrucción.

Es imprescindible la separación del espacio intra-extracraneal y la obliteración del espacio muerto con objeto de disminuir complicaciones infecciosas asociadas a dicho espacio. A la hora de elegir el método reconstructivo es importante considerar los tejidos disponibles, el volumen de espacio muerto extradural predecible, el estado de la duramadre y el pronóstico del paciente.
El uso de tejidos vascularizados, como colgajos locales o libres vascularizados, ha disminuido considerablemente la morbilidad y la mortalidad asociadas ${ }^{11}$.

\section{Conclusiones}

La EDCPCD es una enfermedad rara en la ATM. Las masas tumorales que surgen como tumores primarios en la ATM representan un problema diagnóstico desafiante. La variada presentación de estas lesiones a menudo enmascara la patología de base y conlleva el retraso del diagnóstico. Su tratamiento es eminentemente quirúrgico.

\section{B I B L I O G R A F Í A}

1. Bavitz JB, Chewning LC. Malignant disease as temporomandibular joint dysfunction: review of the literature and report of a case. J Am Dental Asoc. 1990;120:163-6.

2. McCarthy DJ, Kohn NN, Faires JS. The significance of calcium pyrophosphate crystals in the synovial fluid of arthritic patiens: The "pseudogout syndrome". Clinical aspects. Am Intern Med. 1962;56:711-37.

3. Zitnan D, Sitag S. Mnohopcentna familiarha kalcifikaaz artikularnych chrupiek. Bratisl Lek Listy. 1958;38:217-28.

4. Ellman $\mathrm{MH}$, Brown NL, Levin B. Prevalence of knee chondrocalcinosis in hospital and clinic patients aged 50 or older. J Am Geriatr Soc. 1981;29:189-92.

5. Barthelemy I, Karanas Y, Sannajust JP, Emering C, Mondie JM. Gout of the temporomandibular joint: pitfalls in diagnosis. J Craniomax Surg. 2001;29:307-10.

6. Ishida T, Dorfman HD, Bullough PG. Tophaceous pseudogout (tumoral calcium pyrophosphate dihydrate crystal deposition disease). Hum Pathol. 1995;26:587-93.

7. Mostafapour SP, Futran ND. Tumors and tumorous masses presenting as temporomandibular joint syndrome. Otolaryngol Head Neck Surg. 2000;123:459-64.

8. Aoyama S, Kino K, Amagasa T, Kayano T, Ichinose S, Kimijima Y. Differential diagnosis of calcium pyrophosphate dehydrate deposition of the temporomandibular joint. Br J Oral Maxillofac Surg. 2000;38:550-3.

9. Allias-Montmayeur F, Durroux R, Dodart L, Combelles R. Tumours and pseudotumorous lesions of the temporomandibular joint: a diagnostic challenge. J Laryngol Otol. 1997; 111:776-81.

10. Georgantopoulou A, Hodkingson PD, Gerber CJ. Cranial-base surgery: a reconstructive algorithm. Br J Plastic Surg. 2003;56:10-3.

11. Chang DW, Langstein HN, Gupta A, De Monte F, Do KA, Wang X, et al. Reconstructive management of cranial base defects after tumor ablation. Plast Reconstr Surg. 2001;107:1346-55. 